\title{
Vinculando Normas Internacionales de Educación y Tecnologías de la Información para alcanzar el desarrollo del Perú
}

\section{CPC Carlos Alberto Pastor Carrasco}

Docente Auxiliar de la Facultad de Ciencias Contables

\section{RESUMEN}

Este trabajo tiene como objetivo fundamental vincular las normas internacionales emitidas por la Federación Internacional de Contadores (IFAC) con las Tecnologías de Información disponibles en la actualidad en la buisqueda del fortalecimiento de la profesión contable para ofrecer servicios consistentes de alta calidad en beneficio del desarrollo del Perú.

Se analizará el contenido de las Normas Internacionales de Educación (NIE) que tienen por objetivo establecen los elementos esenciales que la educación y los programas de extensión deben de contener y que tienen el potencial para ser reconocidos, aceptados y aplicados internacionalmente.

Se confirmó que si se contrastara lo establecido en las Normas Internacionales de Educación con el modelo en que se utilicen las TIC, se podrá eliminar las brechas más importantes y las perspectivas futuras para alcanzar la convergencia internacional.

La educación debe tener una labor fundamental en el pleno aprovechamiento de las TIC. No se trata simplemente de tener tecnología y condiciones tecnológicas adecuadas, es necesario contar con individuos que puedan darle un uso inteligente e innovador a esa tecnología, permitiendo mejorar y potenciar su uso. La formación de profesionales debe ser acorde con las exigencias del mercado, manteniendo una actualización constante

Palabras clave: Normas Internacionales de Educación, Tecnologías de Información, Formación del Contador Público. 


\section{INTRODUCCIÓN}

La misión de la Federación Internacional de Contadores (IFAC ${ }^{1}$ ) es despertar el interés público, reforzar la profesión de contaduría mundial y colaborar en el desarrollo de las economías internacionales, estableciendo y promoviendo la adhesión a altos patrones de calidad profesionales, promover la convergencia internacional hacia tales padrones y comentar sobre los asuntos de interés público donde la pericia de la profesión es más relevante. La IFAC, a través de sus consejos independientes, desarrolla normas internacionales sobre ética, auditoría y aseguramiento, formación y las normas internacionales de contabilidad del sector público para su aplicación a empresas y profesionales contables.

Por otro lado, el Instituto Americano de Contadores Públicos Certificados (American Institute of Certified Public Accountants $\mathrm{AICPA}^{2}$ ) ha desarrollado el documento denominado "CPA Visión Proyect ${ }^{3}$ ", donde exponen sus ideas sobre la necesidad de la internacionalización de la educación contable. "Por las condiciones de globalización en la contabilidad es conducente prepararse para garantizar: información de mejor calidad, oportuna y comprensible para usuarios de distintos paises; en un lenguaje común que facilite la comparación de información contable entre países y la toma de decisiones en mercados internacionales; capacidad creativa en un ambiente de competitividad; agilidad en los mercados y servicios al cliente; adaptación a las repercusiones de la inversión extranjera, alianzas estratégicas y fusiones de grandes empresas". Representados en la posibles prestación de servicios internacionales, estableciendo algunos parámetros para el éxito del ejercicio de la Contaduría Pública".

El objetivo principal de armonizar las Normas Internacionales de Educación y las tecnologías de la información en el proceso educativo universitario de formación de nuevos profesionales contadores públicos, debe ser el de mejorar el nivel de vida de las personas y desarrollar sus habilidades de contribuir a esa mejoría.

El desarrollo de una nación no se circunscribe a factores competitivos o a indicadores económicos, sino a promover el desarrollo social y económico, a través de la transformación, empoderamiento ${ }^{4}$ y vinculación de las personas, grupos y organizaciones, instituciones y empresas, comunidades, la sociedad y la economía.

\section{Análisis de las Normas Internacionales de Educación (NIE)}

Las NIE tienen por objetivo establecer elementos mínimos que la educación y los programas de extensión universitaria deben contener en su currícula y que debe de tener el potencial de ser reconocidos, aceptados y aplicados en todos los países del mundo. Un objetivo es producir contadores profesionales capaces de hacer una contribución positiva a la profesión y a la sociedad en la que trabajarán a lo largo de su vida.

Las NIE establecen un punto de calificación en el que se deberán evaluar las ca-

1 International Federation of Accountants (IFAC).

2 American Institute of Certified Public Accountants.

3 http://www.aicpa.org/vision/index.htm [en línea] consultado el 23-9-08 15:27.

Empowerment quiere decir potenciación o empoderamiento que es el hecho de delegar poder y autoridad a los subordinados y de conferirles el sentimiento de que son dueños de su propio trabajo. En inglés "empowerment" y sus derivados se utilizan en diversas acepciones y contextos, pero en espańol la palabra se encuentra en pugna con una serie de expresiones que se aproximan sin lograr la plenitud del sustantivo. 
pacidades y competencias mínimas que debe demostrar una persona para ser considerada como un "contador público".

\section{Educación contable}

En ella se hace referencia a los conocimientos, habilidades, actitudes, valores y ética profesional que junto con la experiencia, deben traducirse en las competencias relevantes y apropiadas a las responsabilidades profesionales. Cada universidad debe establecerse estándares cierto nivel de competencia en las siguientes áreas:

a. la educación,

b. la experiencia práctica, y

c. la educación continua

La NIE 1 establece un periodo de precalificación el cual debe de ser suficientemente largo e intensivo para alcanzar las competencias y tener un buen desarrollo profesional en el futuro.

La meta de la educación contable, así como de la experiencia práctica, es producir contadores públicos competentes que sean capaces de hacer una contribución positiva en la profesión y en la sociedad.

La formación debe incidir en el objetivo de que los alumnos tengan claro el concepto de "Aprender a aprender" lo cual involucra el desarrollo de habilidades y estrategias que ayuden a los individuos a aprender más efectivamente y usar estas estrategias para continuar aprendiendo. Es un proceso de entendimiento y de uso de estrategias para mejorar la habilidad de retener y aplicar el conocimiento. P. Senge mencionaba en su obra La Quinta disciplina, que: “...la capacidad de aprender con mayor rapidez que los competidores quizá sea la única ventaja competitiva sostenible".

Asimismo, la educación y experiencia práctica de los contadores públicos debe de otorgar el fundamento del conocimiento, habilidades, valores, ética y actitudes que les permitan continuar adaptándose al cambio a lo largo de sus vidas profesionales.

Estas capacidades les ayudaran a identificar problemas, saber qué conocimiento se requiere para resolver dichos problemas, saber en dónde encontrar este conocimiento, y, finalmente, saber cómo aplicar dicho conocimiento de una manera ética para alcanzar soluciones apropiadas.

La NIE 2 establece que los estudios profesionales de contabilidad deben ser parte del programa de pre-calificación que debe incluir las tres áreas de conocimientos que serán troncos fundamentales:

- Contabilidad,

- Finanzas,

- Conocimiento organizacional y de negocios, y

- Tecnologías de Información (TI)

Destrezas profesionales y educación general (NIE 3), contribuir al desarrollo de las habilidades requeridas por los contadores profesionales, tales como: Habilidades intelectuales, técnicas y funcionales. Personales como el esceptisimo profesional, Habilidades interpersonales y de comunicación, como Trabajar en equipo y Negociar. Habilidades organizacionales y de administración de negocios.

IFAC a través de la NIE 4 -"Valores profesionales, las éticas y las actitudes" reconoce que la profesión del Contador Público en el mundo funciona en ambientes con culturas diferentes y requisitos reguladores. El comportamiento ético es tan importante como las competencias técnicas.

\section{Experiencia práctica contable}

La NIE 5 establece que la experiencia profesional provee de un ambiente propicio 
para desarrollar las competencias requeridas porque: Aumenta el entendimiento sobre las organizaciones, el funcionamiento de los negocios y las relaciones en el trabajo. Y relaciona el trabajo contable con otras funciones $\mathrm{y}$ actividades del negocio.

Los empleadores, colegas de trabajo y consejeros de los aprendices tienen los papeles importantes al planear y monitorear la experiencia práctica adquirida por aprendices.

\section{Capacidades y competencias}

En la NIE 6 se prescribe que al final de las capacidades profesionales y competencias, se tenga conocimientos de Resolución de problemas complejos, Comunicación efectiva de recomendaciones de manera lógica y concisa, e Identificación de dilemas éticos.

\section{Desarrollo continuo}

La NIE 7, en relación al desarrollo continuo, propone promover un compromiso para aprender durante de toda la vida entre los contadores profesionales

La competencia profesional se desarrolla mediante la combinación de estudio académico, capacitación en el trabajo, experiencia laboral y educación profesional. El enfoque académico cubrirá la teoría contable, mientras que la educación profesional se enfocará más en la práctica y las habilidades que necesitan los contadores profesionales, la experiencia laboral se concentrará en la capacitación en el trabajo y en la actualización continua.

\section{Especializaciones profesionales}

El ligar los tres enfoques (académico, educación profesional y experiencia laboral) para desarrollar competencias funcionales y capacidades será más productivo que el intentar desarrollar una amplia gama de competen- cias y capacidades requeridas por un solo camino.

\section{Norma 11}

EL IFAC en su Norma 11 "Education Committee November 1996 Implementing International Education Guideline 11 Information Technology in the Accounting Curriculum" identifica los requerimientos de educación de la Tecnología de la Información para el Contador Público dentro de cinco encabezados principales:

- Los requisitos generales en educación de tecnología de Información.

- El contador como usuario de TI.

- El contador como administrador en sistemas de información.

- El contador como diseñador de sistemas gerenciales.

- El contador como evaluador de sistemas de información.

\section{RESULTADOS}

\section{Tecnologías de Información aplicadas a la} Contabilidad

La universidad del siglo XXI, como institución mediadora entre los conocimientos y las personas, puede favorecer o limitar el desarrollo personal y colectivo.

Frente a las nuevas demandas sociales ligadas a los procesos de globalización, la universidad del siglo XXI debe abordar nuevas formas de mediación. En este sentido, el uso que se asigne a las nuevas tecnologías de información y comunicación pueden concretar nuevas formas de mediación posibilitando cambios en la enseńanza, en las condiciones de aprendizaje y en las situaciones evaluativas. Ellas deben modificar los roles del que aprende, del que enseña y del ambiente de trabajo universitario. 
Surgió la idea de proponer a la Comisión Permanente de Evaluación Curricular y Coordinación Académica, modificar los Planes de Estudios de anual a semestral, en donde los tres primeros años sean de Estudios Generales, y los dos últimos de especialización. Con la meta final de graduar a los futuros Contadores Públicos, con mención en una especialidad: Auditoría, Contabilidad, Costos y Finanzas y Tributación. Idea que aún ninguna universidad, pública o privada, lo ha planteado a nivel nacional.

Mediante Ley No 28951, se expide la Ley de Actualización de la Ley No 13253 de Profesionalización del Contador Público y de creación de los Colegios de Contadores Públicos, y en su Artículo $3^{\circ}$ se establecen las competencias del contador público, en las que todas las actividades indicadas tienen relación con TI.

Asimismo, en el documento "Perfil del Contador Público" se establece como un objetivo de la carrera: "El diseño de sistemas de información y decisión para el logro de los objetivos de la organización; sistemas de registro e información contable; sistemas de control de gestión y auditoría operativa”.

\section{Los requerimientos generales en educación de Tecnología de Información:}

Todo contador debe adquirir los siguientes conocimientos esenciales de TI relacionados con los sistemas gerenciales:

- Conceptos de tecnología de la información para sistemas gerenciales.

- Control interno en sistemas de negocios computarizados.

- Administración de la adopción de TI y su uso.

- Estándares de desarrollo y práctica para sistemas administrativos.

- Evaluación de sistemas administrativos basados en computadoras.
Conceptos de tecnología de la Información para sistemas gerenciales

Los temas que deben ser incluidos serán los siguientes: Conceptos de TI para sistemas gerenciales, Uso de la Administración de Información, Hardware, Sistema de software, Software de aplicación, Organización de datos y métodos de acceso, Redes y transferencia electrónica de datos, Procesamiento de transacciones en aplicaciones típicas administrativas y de Contabilidad.

Control Interno en Sistemas de negocios computarizados

El control referido a objetivos, Marco de referencia del control, Medio Ambiente de control, Asignación de riesgo y Control de actividades

Administración de la adopción de TI y su uso Consideraciones estratégicas en el desarrollo de tecnología de la información, Problemas administrativos, Control financiero sobre la TI, Problema de Operación, Seguridad, respaldo y recuperación.

Estándares de desarrollo y práctica para sistemas administrativos

- Papel de la información en el diseño de la organización y su comportamiento

- Técnicas de diseño de sistemas

- Fases del ciclo de vida de adquisición/ desarrollo de sistemas y control del mantenimiento sobre el proceso de desarrollo.

Evaluación de sistemas administrativos basados en computadoras

- Legal, ético, auditoría y estándares de sistemas de control

- Objetivos de evaluación

- Técnicas de auditoría asistida por computadora (CAATS) 


\section{Usuario de TI}

Como usuario de la tecnología del información, los contadores deberían además conocer cómo probar y aceptar un sistema en particular que fue autorizado o desarrollado y como operarlo y mantenerlo el día.

Los contadores deberán tener los conocimientos de procesos básicos usados para mantener los recursos de sistemas organizados, y un control de los procesos y prácticas para salvaguardar sus sistemas y datos en contra de los errores, así como evitar el uso autorizado, la piratería de software y los ataques de virus, vandalismos y falla del sistema.

\section{Administrador en Sistemas de Información}

Para mantener su papel de jefes de sistemas de información, los contadores deberán tener un buen entendimiento de las funciones de la administración en los sistemas de información para que puedan cumplir con este fin y con los procesos administrativos relacionados con la coordinación y planeación, organización, personal, dirección y liderazgo, control y comunicación en un contexto de uso de la tecnología de información.

\section{Diseñador de sistemas gerenciales}

Los contadores, como empleados consultores externos, han estado involucrados en el diseño y sistemas financieros por décadas. En el pasado, los papeles de diseño habían estado en el contexto de sistemas que serían para guardar registros de forma manual. Hoy en día, los contadores esperan seguir proporcionando servicios similares, aunque un contexto de tecnología de información. Esto podría ser como miembro del equipo de oficina o fuerza de trabajo para establecer requerimientos de sistema de negocios, como miembro del equipo de desarrollo dentro del sistema local para un empleo o como un consultor externo ayudando dicen ya un sistema administrativo para un cliente.

Los contadores deberán estar conscientes de los estándares y prácticas utilizadas, particularmente prácticas de control interno, que podrían guiar las prácticas de diseño de sistemas de información.

El conocimiento de los contadores o sistema de información deberá ser desarrollado en un contexto de ganar y entender las por la organización de los negocios y los objetivos de servicio y sus ambientes. Así, los programas de educación y cursos que aspiran a desarrollar el conocimiento del diseño sistemas deberán tener un sentido administrativo en vez de una eventración técnica.

\section{Evaluador de sistemas de información}

El papel del contador, como evaluador, comprende la función de auditor interno, el auditor externo y otros papeles de evaluación realizados por los contadores, ya sean identificados como papeles del auditor.

Todo los contadores involucrados en el papel de evaluación en el momento de la precalificación deben tener la habilidad, como una limitada supervisión, de planear, ejecutar y comunicar los resultados obtenidos para un tipo específico de valuación para su área de trabajo en el contexto de circunstancias específicas que involucra sistemas de información.

\section{Nuevo entorno de la transmisión del conocimiento contable para el desarrollo sostenible}

La educación, de acuerdo a las normas internacionales, tiene un papel fundamental en el desarrollo y crecimiento económico, no solo promueve el desarrollo, también potencia la reducción de desigualdades sociales y mejora el nivel de vida de la sociedad en general. El acceso libre y universal a 
la educación es vital para lograr estas metas; sin embargo, en la actualidad no basta con esto, es necesario que en la educación se garantice la calidad. En este sentido, el Estado juega un papel fundamental en la búsqueda de nuevos métodos e instrumentos que contribuyan a fortalecer los procesos de enseñaza y aprendizaje.

Así como las Tecnologías de Información y Comunicación (TIC) tienen un impacto significativo en la formación del contador público, también pueden contribuir, no solo al crecimiento económico, como resultado de mejoras en la productividad y reducción de costos, sino, también, a potenciar el desarrollo e incrementar la disponibilidad de conocimiento para los procesos de toma de decisiones. La inversión en capital y tecnología es un factor importante por considerar, pero no el único, debe ir acompañado por una fuerte inversión en capital humano paralelo a una implementación estratégica de las TIC.

Sobre el ámbito de la enseñanza los beneficios de las TIC suponen:

- Una mayor interacción entre estudiante y profesores, en especial mediante la utilización del correo electrónico, de la videoconferencia y de la WWW;

- una más intensa comunicación entre estudiantes, mediante grupos de trabajo y de discusión que se apoyen en las nuevas herramientas comunicativas;

- un mejor aprendizaje mediante el uso de simuladores;

- el desarrollo de competencias y de habilidades prácticas por parte de los estudiantes en laboratorios virtuales de investigación;

- la provisión de posibilidades de retroacción en la comunicación entre los estudiantes; el acceso de los estudiantes a recursos educativos.

\section{CONCLUSIONES}

Desde nuestro punto de vista, los efectos van a ser mucho mayores, ya que estamos transitando no solo entre modelos tecnológicos distintos, sino que discurrimos entre modelos gramaticales distintos y, por tanto, como cualquier semiólogo podría aventurar, esto tiene efectos profundos sobre las formas del conocimiento.

La traslación de estas ventajas al marco de la enseñanza universitaria no es del todo automática ya que el marco universitario no solo trata de convertir información en conocimiento instrumental, sino que las misiones y funciones a cumplir por la universidad amplían notablemente el catálogo, y entre ellas podemos destacar: servir a la sociedad que la sustenta, contribuir al desarrollo socioeconómico de su entorno, posibilitando, además, una mayor generación de valor añadido y de riqueza al sistema empresarial y al conjunto de la sociedad, ofrecer iniciativas y abrir proyectos a su entorno social, divulgar una cultura cívica, técnica y artística, crear y potenciar una conciencia y capacidad de trabajo, basada en la eficacia, la independencia, la profesionalidad, la optimización de recursos y la autocrítica.

Dadas las exigencias y transformación que enfrenta la sociedad actual, las universidades deben desarrollar profesionales con las habilidades y destrezas para el uso inteligente e innovador de las TIC, de forma tal que los nuevos profesionales puedan contribuir con un verdadero desarrollo sostenible. Mejores profesionales en este aspecto, permiten mejorar las condiciones de empleo, la atracción de inversiones que generen valor agregado, el incremento de nuestra productividad, avances en la calidad de vida entre otros factores del desarrollo de una sociedad. 
La adopción de las normas internacionales en el Perú podría generar los siguientes impactos:

a. En el campo de la educación. Las universidades deberán enfocarse en el desarrollo de las competencias requeridas por la profesión enfatizando en los siguientes aspectos:

- Desarrollo de temarios integrales, con enfoque en la educación general y el desarrollo de habilidades y competencias.

- Técnicas enfocadas en lograr un aprendizaje continuo.

- Uso de casos, proyectos, simulaciones y grupos de trabajo.

- Entorno de clases con recreación de ambientes de trabajo.

- Uso intensivo de tecnología.

- Intercambio de profesores entre países desarrollados y en desarrollo.

b. En el campo del desarrollo económico. Deberá generar una mayor certeza a los inversionistas en relación a sus inversiones, ya que existirán normas de registro estandarizadas en base a las mejores prácticas internacionales, lo cual garantizará la comparabilidad de cifras entre países.

c. En el campo del entorno regulatorio. Estará en condiciones de ofrecer al inversionista nacional e internacional, un conjunto de normas que reflejen la operatividad real de las empresas bajo un estándar internacional que respeta las diferencias entre los distintos países $y$ que favorece el intercambio de información de las distintas agencias reguladores tanto a nivel nacional como internacional.

\section{REFERENCIAS}

Arnaz A., José. La planeación curricular. Edición Trillas. 1989.

Casalet, Mónica; Casas Rosalba. Un diagnóstico sobre la vinculación Universidad-Empresa. Conacyt-Anuies (Serie Investigaciones) International Education Standards del IFAC

http://es.ifac.org/Store/Category. tmpl?Category=Education

Echenique García, José Antonio. La tecnología de la información en la currícula del contador. IMCP-IFAC, México. Mayo 2003. Primera Edición.

Gore, Al. Una verdad incómoda. Editorial Gedisa, España, 2007, Primera edición.

Jimeno Sacristán, J. y A. I. Pérez G. Comprender y transformar la enseñanza. Ed. Morata. S. L. Madrid, 1999.

Nerici, Imideo G. Metodología de la enseñanza. Editorial Kapelusz, México D.F., 1984.

Ruiz Durán, Clemente. El reto de la educación superior en la sociedad del conocimiento. ANUIES Libros en línea.

Senge, Peter M. La quinta disciplina. Argentina, 1998.

Toffler, Alvin. La tercera ola. Plaza \& Janes. España, 1981.

Udual. Administración Universitaria en América Latina. Santiago de Chile, 1995. 